\title{
Das neue Strahlenschutzgesetz - Auswirkungen auf die radioonkologische Forschung in Deutschland
}

\author{
Torsten Nölling' \\ Online publiziert: 22. Dezember 2017 \\ ○) Springer-Verlag GmbH Germany, part of Springer Nature 2017
}

\section{Zusammenfassung}

Vor dem Hintergrund der Europäischen Richtlinie 2013/59/Euratom hat der Bundesgesetzgeber das neue Strahlenschutzgesetz erlassen. Dieses wird ab dem 01.01.2019 die Anwendung ionisierender Strahlung in der Heilbehandlung und der medizinischen Forschung regeln. Während die grundsätzliche Aufteilung zwischen Heilbehandlung und Forschung beibehalten wird, offenbaren sich im Detail mitunter gravierende Unterschiede zum bestehenden Recht. Die Neuregelungen für den Bereich der radioonkologischen Forschung werden in diesem Beitrag beleuchtet. Der Fokus liegt dabei auf der Darstellung der neuen Fristenregelungen für die Genehmigung durch bzw. die Anzeige bei der zuständigen staatlichen Aufsichtsbehörde. Dabei wird deutlich, dass es gelungen ist, eine klare Verbesserung der bisher für die Forschenden unbefriedigenden Rechtslage zu erreichen: Mit dem Ziel die Wettbewerbsfähigkeit des Forschungsstandorts Deutschland nicht weiter zu gefährden, wurde auf den letzten Metern des Gesetzgebungsverfahrens noch die wichtige Fristenregelung bei der Genehmigung radioonkologisch-therapeutischer Forschungsvorhaben erreicht, die zu einer nachhaltigen Beschleunigung der Genehmigungspraxis in Deutschland führen dürfte.

\section{Hintergrund}

Der Gesetzgeber ist mit dem „Gesetz zur Neuordnung des Rechts zum Schutz vor der schädlichen Wirkung ionisierender Strahlung" seiner europarechtlichen Verpflichtung zur Umsetzung der Richtlinie 2013/59/Euratom des Rates (EUGrundnorm) in nationales Recht nachgekommen. Wichtigster Teil dieses Gesetzes ist das neue Strahlenschutzgesetz (StrlSchG) ${ }^{1}$, welches ab 2019 das bisherige Strahlenschutzrecht, das insbesondere in der Strahlenschutzverordnung und der Röntgenverordnung enthalten ist, ersetzen wird. Wie auch bisher handelt es sich bei dem neuen Strahlenschutzrecht zunächst um Gefahrenabwehrrecht. Ziel des Gesetzes sind nach $\S 1$ Abs. 1 StrlSchG „Regelungen zum Schutz des Menschen (...) vor der schädlichen Wirkung ionisierender Strahlung (...)“. Der im bisherigen Recht existierende Zielkonflikt, radioonkologische Thera-

\footnotetext{
${ }^{1}$ Gesetz zum Schutz vor der schädlichen Wirkung ionisierender Strahlung (Strahlenschutzgesetz - StrlSchG), veröffentlicht im BGB1 vom 03.07.2017, S. 1966-2067.
}

$\triangle$ Torsten Nölling

Post@RA-Noelling.de

1 Rechtsanwalt - Fachanwalt für Medizinrecht, Rödelstraße 13, 04229 Leipzig, Deutschland pien und insbesondere auch radioonkologische Forschung zu ermöglichen und zugleich Menschen vor der schädlichen Wirkung ionisierender Strahlung zu schützen, besteht damit auch unter dem neuen StrlSchG fort.

\section{Die Beteiligung der Behörde bei Forschungsvorhaben in der Strahlentherapie}

Das neue Strahlenschutzgesetz sieht zwingend eine Beteiligung der zuständigen Strahlenschutzbehörde vor, wenn im Rahmen eines Forschungsvorhabens ionisierende Strahlung $\mathrm{zu}$ diagnostischen oder therapeutischen Zwecken angewendet werden soll. Die zuständige Behörde wird nach § 185 Abs. 1 Nr. 1 und 2 StrlSchG das Bundesamt für Strahlenschutz (BfS) sein. Insofern entspricht das neue Strahlenschutzrecht dem alten.

\section{Genehmigungs- und Anzeigeverfahren} (§§ 31, 32 StrlSchG)

Auch die Differenzierung bei der Intensität der Beteiligung je nach Art des Forschungsvorhabens bleibt bestehen. Hier wird jedoch eine neue Nomenklatur eingeführt. Nach der bisherigen Rechtslage differenzieren $\S \S 23,24$ StrlSchV zwischen dem umfangreichen ,normalen“ Genehmigungsverfahren nach $\S \S 23,24$ Abs. 1 StrlSchV und dem ,,ver- 
einfachten" Genehmigungsverfahren nach $\S \S 23,24$ Abs. 2 StrlSchV. Während Ersteres bei Forschungsvorhaben mit geplantem therapeutischem Einsatz ionisierender Strahlung durchlaufen werden muss, ist Letzteres solchen Forschungsvorhaben vorbehalten, die ionisierende Strahlung allein zu begleitdiagnostischen Zwecken einzusetzen beabsichtigen.

Das neue StrlSchG differenziert nunmehr zwischen einer Genehmigung nach $\S 31$ StrlSchG und einer Anzeige nach $\S 32$ StrlSchG. Auch die Aufteilung der Forschungsvorhaben wird im Grundsatz beibehalten. Der therapeutische Einsatz von ionisierender Strahlung bedarf auch zukünftig einer Genehmigung durch das BfS nach $\S \S 31,185$ Abs. 1 Nr. 2 StrlSchG. Bei allein begleitdiagnostischem Einsatz ionisierender Strahlung wird zukünftig ein Anzeigeverfahren beim BfS nach $\S \S 32,185$ Abs. 1 Nr. 2 StrlSchG an die Stelle des bisherigen vereinfachten Genehmigungsverfahrens treten. Der Anwendungsbereich des Anzeigeverfahrens wird im Vergleich zum vereinfachten Genehmigungsverfahren etwas umfangreicher sein und auch Forschungsvorhaben umfassen, die nicht einwilligungsfähige Personen einschließen. ${ }^{2}$

Bevor der Fokus auf die jeweiligen Verfahrensabläufe und die weitergehende Differenzierung zwischen dem Genehmigungsverfahren nach $\S 31 \mathrm{StrlSchG}$ und dem Anzeigeverfahren nach $\S 32$ StrlSchG gelegt werden wird, soll zunächst der Anwendungsbereich der forschungsspezifischen Regulierungsvorschriften in Abgrenzung zur Heilbehandlung geklärt werden.

\section{„Medizinische Forschung“ im Sinne des Gesetzes}

Entscheidend für die Reglementierung der radioonkologischen Forschung unter dem neuen Strahlenschutzgesetz wird der Geltungsbereich der entsprechenden Vorschriften sein. Wie auch bisher in der Strahlenschutzverordnung unterscheidet das StrlSchG zwischen der Anwendung ionisierender Strahlen zu Heilzwecken (Kap. 5, §§ $83 \mathrm{ff}$. StrlSchG) und zum Zwecke der medizinischen Forschung (Kap. 2 und 5. Abschnitt, §§ 31-37 StrlSchG). Da bei strahlentherapeutischen Forschungsvorhaben allein erkrankte Probanden als Studienteilnehmer in Betracht kommen, werden - wie bisher - sowohl die Vorschriften über den Einsatz von ionisierenden Strahlen zu Heilzwecken als auch die Vorschriften zur medizinischen Forschung anzuwenden sein. Der Begriff „,medizinische Forschung“ wird in $\S 5$ Abs. 23 StrlSchG legal definiert und ist in seiner strahlenschutzrechtlichen Bedeutung enger als im Allgemeinen medizinischen Verständnis.

Medizinische Forschung im Sinne des neuen Strahlenschutzgesetzes ist die

2 BT-Drs. 18/11241, S. 272.
Fortentwicklung medizinischer Untersuchungsmethoden, Behandlungsverfahren oder der medizinischen Wissenschaft. Medizinische Forschung liegt nicht vor, wenn die Anwendung radioaktiver Stoffe oder ionisierender Strahlen ausschließlich der Untersuchung oder Behandlung der einzelnen Person dient.

Nach der bisher geltenden Rechtslage wird der Begriff „Medizinische Forschung“ leicht anders gefasst. § 3 Abs. 2 Nr. 14 StrlSchV definiert:

\section{Forschung, medizinische:}

Die Anwendung radioaktiver Stoffe oder ionisierender Strahlung am Menschen, soweit sie der Fortentwicklung der Heilkunde oder der medizinischen Wissenschaft und nicht in erster Linie der Untersuchung oder Behandlung des einzelnen Patienten dient.

In der Gesetzesbegründung wird betont, dass es sich allein um eine sprachliche Anpassung handele, mit der keine Änderung der Bedeutung einhergehen soll. ${ }^{3}$

Die Frage, welche Anwendung von ionisierender Strahlung allein als Heilbehandlung im Sinne des $§ 83$ StrlSchG und welche (auch) als medizinische Forschung im Sinne der $\S \S 31 \mathrm{ff}$. StrlSchG gelten wird, ist für die tägliche Praxis von höchster Relevanz. In der Vergangenheit wurde in einem langwierigen und intensiven Austausch zwischen den interessierten Kreisen und unter Zugrundelegung der bisher geltenden Rechtslage nach StrlSchV ein Instrumentarium zur Abgrenzung von Heilbehandlung, die auch den individuellen Heilversuch umfasst, zu medizinischer Forschung erstellt und vereinbart. ${ }^{4}$ Dieser erreichte Konsens ist durch die neue Legaldefinition gefährdet.

Bei einer genaueren Betrachtung der Gesetzgebungshistorie fällt auf, dass es eine Änderung des Normtextes gab. Während der Referentenentwurf vom 14.09.2016 ${ }^{5}$ die Formulierung des $\S 3$ Abs. 2 Nr. 14 StrlSchV weitestgehend übernahm und insbesondere die Formulierung ,und

\footnotetext{
3 BT-Drs. 18/11241, S. 229.

4 Simon et al: Genehmigungsverfahren klinischer Studien im Bereich der Radioonkologie, Strahlenter Onkol (2015) 191: 909-920; $B f S$; Hinweise zu Anträgen auf Genehmigung der Anwendung von radioaktiven Stoffen, ionisierender Strahlung und Röntgenstrahlen am Menschen in der medizinischen Forschung nach $\$ 23$ StrlSchV bzw. $\S 28$ a RöV (abrufbar unter www.bfs.de); BfS; Neuartige Strahlenanwendungen in der Medizin: Abgrenzung Heilkunde - Medizinische Forschung Ergebnisse eines BfS-Fachgesprächs am 21.03.2013 (abrufbar unter: http://www.bfs.de/DE/themen/ion/anwendung-medizin/ forschung/hinweise/hinweise_node.html)

5 Referentenentwurf des Bundesministeriums für Umwelt, Naturschutz und Reaktorsicherheit - Entwurf eines Gesetzes zur Neuordnung des Rechts zum Schutz vor der schädlichen Wirkung ionisierender Strahlung vom 14.09.2016; abrufbar unter: http://www. atommuellkonferenz.de/wp-content/uploads/Referentenentwurf_ Strahlenschutzgesetz_Ressortabstimmung-2016-09-14.pdf (Stand: 16.08.2017).
} 
nicht in erster Linie der Untersuchung oder Behandlung der einzelnen Person dient " fortführte, wurde diese Formulierung bis zur Veröffentlichung des Kabinettsentwurfs und damit der Regierungsvorlage in die nunmehr in $\S 5$ Abs. 23 StrlSchG Gesetz gewordene Fassung verändert. Die jeweilige Begründung ist hingegen nicht geändert worden. In der Begründung zum Referentenentwurf (v. 14.09.2016, S. 216) heißt es:

Bislang wurde der Begriff „,medizinische Forschung“ in $\$ 3$ Absatz 2 Nummer 14 der bisherigen Strahlenschutzverordnung und $\$ 2$ Nummer 8 der bisherigen Röntgenverordnung bestimmt. Die in den bisherigen Begriffsbestimmungen verwendeten Wörter „,der Heilkunde“ werden durch die Wörter „medizinischer Untersuchungsmethoden und Behandlungsverfahren “ ersetzt. Eine inhaltliche Änderung des Begriffs medizinische Forschung ist damit nicht beabsichtigt.

Die Gesetzesbegründung zu $§ 5$ Abs. 23 StrlSchG (BTDrs. 18/11241, S. 229) formuliert:

Die Definition greift die Begriffsbestimmungen „Forschung, medizinische "in \$ 3 Absatz 2 Nummer 14 der bisherigen Strahlenschutzverordnung und $\$ 2$ Nummer 8 der bisherigen Röntgenverordnung auf. Unter anderem werden die dort verwendeten Wörter ,,der Heilkunde“ durch die Wörter „,medizinischer Untersuchungsmethoden, Behandlungsverfahren" ersetzt; eine inhaltliche Änderung des Begriffs medizinische Forschung ist damit nicht beabsichtigt.

Während die Gesetzesbegründung damit unverändert betont, dass durch die Änderung des Wortlauts der Vorschrift keine inhaltliche Änderung beabsichtigt sei, wurde der Wortlaut an entscheidender Stelle geändert. Es macht rechtlich und tatsächlich einen Unterschied, ob die Anwendung der ionisierenden Strahlung in erster Linie oder ausschließlich der Untersuchung oder Behandlung der einzelnen Person dient.

Es ist damit zu konstatieren, dass es dem Gesetzgeber nicht gelungen ist, seinen Willen, eine inhaltliche Änderung des Begriffs ,medizinische Forschung“ nicht vorzunehmen, eindeutig in den Gesetzestext einzubringen. Aus juristischer Sicht kommt dem Willen des Gesetzgebers aber im Vergleich zum Wortlaut des Gesetzes nur eine untergeordnete Bedeutung zu. Die Gerichte sind an das Gesetz gebunden, nicht an die Gesetzesbegründung. Bei einem solch offensichtlichen Missverhältnis zwischen Wortlaut der Norm und Intention des Gesetzgebers ist die Gesetzesbestimmung nach allgemeinen juristischen Methoden auszulegen. Neben der (strengen) Wortlautauslegung existieren gleichberechtigt weitere Auslegungsmethoden. Zunächst zu nennen ist die historische Auslegung, die insbesondere die Gesetzesbegründung heranzieht und damit den (historischen) Wil- len des Gesetzgebers bemüht. Gleichberechtigt kommen die systematische Auslegung, also die Auslegung nach dem $\mathrm{Zu}-$ sammenhang in dem die Norm steht, und die teleologische Auslegung, also die Frage nach Sinn und Zweck der Vorschrift, zum Tragen. Schließlich ist die Norm im Einklang mit der zugrundliegenden europäischen Grundnorm ${ }^{6}$ auszulegen.

\section{Wortlautauslegung und der Wille des Gesetzgebers}

Der Anwendungsbereich der Vorschriften über medizinische Forschung ist mit der neuen Formulierung größer geworden. Immer dann, wenn die Anwendung der ionisierenden Strahlung auch der ,Fortentwicklung medizinischer Untersuchungsmethoden, Behandlungsverfahren oder der medizinischen Wissenschaft “ (im Folgenden: wissenschaftliche Erkenntnis) dient, sind nach dem reinen Wortlaut des $\S 5$ Abs. 23 StrlSchG die Vorgaben der $§ \S 31-37$ StrlSchG und damit die umfangreichen Vorgaben zur Genehmigung oder jedenfalls Anzeige eines Forschungsvorhabens einschlägig. Praktische Bedeutung erlangt diese Differenzierung insbesondere beim Heilversuch, bei retrospektiven Studien, aber auch bei prospektiven Studiendesigns, die etablierte Behandlungsmethoden vergleichen. In diesen Fällen gilt bisher, dass die Anwendung ionisierender Strahlung in erster Linie der Behandlung der Patienten gilt, da die jeweiligen Personen auch ohne das Forschungsvorhaben die jeweilige Behandlung in identischer Form erhielten, und damit keine Genehmigung durch das BfS erforderlich ist. ${ }^{7}$ Bei einer strengen Anwendung der neuen Definition gilt jedoch, dass die Personen die Behandlung nicht ausschließlich zum Zwecke der Behandlung erhalten. Ein (Neben-)Zweck ist auch die wissenschaftliche Erkenntnis.

Dieses Ergebnis ist unbefriedigend und konterkariert die Intention des Gesetzgebers, radioonkologische Studien in Deutschland zukunftssicher zu ermöglichen und bestehende Wettbewerbsnachteile im internationalen Kontext abzubauen. ${ }^{8}$

Während bei einem individuellen Heilversuch - je nach Definition - bei strenger Anwendung des neuen Gesetzeswortlautes vertreten werden kann, dass der Heilversuch ausschließlich der Heilbehandlung dient, ist diese Begrenzung bei retrospektiven Studien bereits schwieriger und bei prospektiven Studien, die etablierte Behandlungsschemata miteinander (randomisiert) vergleichen, nicht mehr haltbar.

\footnotetext{
${ }^{6}$ EU-Grundnorm; RiLi2013-59-Euratom vom 05.12.2013.

7 So das BfS in seinen Hinweisen, a. a. O., S. 2f und Simon et al., Genehmigungsverfahren, a. a. O. S. 913.

8 Stellungnahme des Bundesrates vom 10.03.2017, BR-Drs. 86/17, S. 13; Empfehlungen der Ausschüsse zu Punkt 15 der 954. Sitzung des Bundesrates am 10. 03.2017, BR-Drs. 86/1/7, S. 15.
} 
Denn jedenfalls bei Letzteren erfolgt die Exposition auch zum Zwecke der medizinischen Forschung.

Diese strenge Wortlautauslegung stellt die Ärzte in der Praxis mitunter vor ein medizinrechtlich-ethisches Dilemma. Einerseits sind sie ihren Patienten gegenüber verpflichtet, alles medizinisch Mögliche zu unternehmen, um diese $\mathrm{zu}$ kurieren oder jedenfalls das Leiden zu lindern - bei Fehlen oder Unwirksamkeit einer etablierten Behandlungsmethode auch im Wege des Heilversuchs. Andererseits verlangt das neue Strahlenschutzrecht bei strenger Wortlautauslegung auch in diesen Fällen die Einholung einer Genehmigung nach $\S 31$ StrlSchG. Der damit verbundene Zeitverlust würde in der Praxis dazu führen, dass Heilversuche faktisch nicht mehr stattfinden könnten. Bei retrospektiven Studien und bei prospektiven Studien, die etablierte Schemata vergleichen, besteht dieses Dilemma zwar nicht, denn die Patienten sollen in jedem Fall mit einem etablierten Behandlungsschema therapiert werden. Jedoch führt die strenge Wortlautauslegung dazu, dass entgegen der Intention einer Stärkung des Forschungsstandorts tatsächlich eine weitreichende Schwächung erzielt werden würde. Studiendesigns, die nach aktueller Rechtslage keiner Genehmigung durch das BfS bedürfen, müssten ab 2018 eine solche erhalten, mit allen damit verbundenen Kosten und Zeitverlusten.

\section{Teleologische und europarechtskonforme Auslegung}

Das Strahlenschutzgesetz dient - wie das gesamte europäische und nationale Strahlenschutzrecht - dem Schutz des Menschen vor der schädlichen Wirkung ionisierender Strahlung ( $§ 1$ Abs. 1 StrlSchG). Insbesondere die Vorschriften zur zulässigen medizinischen Exposition stellen im Einklang mit Art. 2 Grundgesetz (GG) - klar, dass dieser Schutz nicht so weit gehen darf und soll, Patienten die Segnungen der ionisierenden Strahlen vorzuenthalten.

Nach zutreffender Ansicht des (historischen) Gesetzgebers stellt „,er Heilversuch (...) einen Sonderfall der Heilbehandlung dar, da hierbei noch nicht abschließend erprobte Methoden oder Mittel mangels anderer erfolgversprechender Methoden oder Mittel vom Arzt mit dem konkreten Ziel einer individuellen Heilmaßnahme angewendet werden." Im Fokus der Behandlung und deren alleiniger Primärzweck (,,in erster Linie“) ist damit die Behandlung des Patienten. Ein etwaiger wissenschaftlicher Erkenntnisgewinn kann allein Sekundärzweck sein und ist nicht wesensbestimmend. Ein individueller Heilversuch beabsichtigt keinen Erkenntnisgewinn über den Einzelfall hinaus; ihm liegt weder ein wissenschaftlicher

\footnotetext{
${ }_{9}$ Begründung zur Novelle des Strahlenschutzrechts 2001, abrufbar unter http://www.bmu.de/service/publikationen/downloads/details/ artikel/strahlenschutzverordnung-verordnung-ueber-den-schutz-vorschaeden-durch-ionisierende-strahlen/.
}

Prüfplan zugrunde, noch besteht die Absicht zur Änderung des Therapiestandards. ${ }^{10}$ Faktisch existiert der Sekundärzweck des wissenschaftlichen Erkenntnisgewinns bei jeder Behandlung eines Patienten, egal ob mit Standarttherapie oder anderweitig. Sollten im Laufe der Behandlung unerwartete Erkenntnisse gewonnen werden, wird der Arzt diese Erkenntnis, z.B. über ein CIRS-System (Critical Incident Reporting System) oder eine UAW-Meldung (unerwünschte Arzneimittelwirkung) oder ggf. im Rahmen einer wissenschaftlichen Veröffentlichung (z. B. als Fallstudie), bekannt machen. Dennoch gilt in diesen Fällen auch nach bisheriger strahlenschutzrechtlicher Wertung die Behandlung nicht als Forschung.

Die EU-Grundnorm schließlich definiert den Begriff „medizinische Forschung“ nicht. Für die Beantwortung der hier relevanten Frage ist sie unergiebig.

\section{Empfehlung}

Aus dieser Erkenntnis folgt, dass die im Gesetzgebungsprozess aus hier unbekannten Gründen erfolgte Änderung des Wortlautes des $\S 5$ Abs. 23 StrlSchG von ,in erster Linie“ zu ,ausschließlich“ bei Beibehaltung der Gesetzesbegründung, nach der eine Änderung der Begriffsbedeutung nicht beabsichtigt sei, im Wege der teleologischen Reduktion dahingehend ausgelegt werden muss, dass auch weiterhin Heilversuche und Forschungsvorhaben, die allein den Vergleich bestehender Standardtherapien zum Ziel haben, nicht dem Begriff der medizinischen Forschung im Sinne des $\S 5$ Abs. 23 StrlSchG unterfallen. $§ 5$ Abs. 23 Satz 2 StrlSchG ist daher wie folgt zu lesen:

\section{Medizinische Forschung liegt nicht vor, wenn die An- wendung radioaktiver Stoffe oder ionisierender Strah- len in erster Linie der Untersuchung oder Behandlung der einzelnen Person dient.}

Unter Berücksichtigung der bis zum geplanten Inkrafttreten des $\S 5$ Abs. 23 StrlSchG am 01.01.2018 verbleibenden Zeit, sollte die Gesetzesformulierung wieder in die ursprünglich bestehende Fassung des Referentenentwurfs geändert werden, um rechtliche Unsicherheiten für die Beteiligten und auch eine unnötige Arbeitsbelastung beim BfS zu vermeiden.

\section{Weitere forschungsrelevante Begriffe des neuen Strahlenschutzrechts}

Auch an anderer Stelle führt das neue Strahlenschutzgesetz neue Begriffe ein. Dies ist zum einen auf die zugrundeliegende europäische Richtlinie, zum anderen auf sprachliche Optimierungen zurückzuführen.

\footnotetext{
${ }_{10}$ Simon et al., Genehmigungsverfahren, a. a. O. S. 915.
} 
Teilnehmer an strahlentherapeutischen Forschungsvorhaben werden zukünftig als (in das Forschungsvorhaben eingeschlossene) „Person“ bezeichnet ( $\$ 2$ Abs. 8 Nr. 2 StrlSchG). ${ }^{11}$ Nach der bisherigen Rechtslage galt der Begriff „Proband“ ( $\$ 3$ Abs. 2 Nr. 32 b StrlSchV), differenzierend nach gesundem ( $\$ 3$ Abs. 2 Nr. 24 a StrlSchV) und ,normalem“, d.h. krankem Probanden. Inhaltlich ändert sich durch diese sprachliche Anpassung nichts. Insbesondere das abweichende Vokabular für Studien nach dem Arzneimittelgesetz (AMG) oder Medizinproduktegesetz (MPG), die jeweils zwischen „Patienten“ (kranken Teilnehmern) und „Probanden“ (gesunden Teilnehmern) unterscheiden, bleibt bestehen.

\section{Genehmigungsverfahren (nach §§ 31, 37 StrISchG)}

Das Genehmigungsverfahren nach $\S \S 31,37$ StrlSchG tritt an die Stelle des bisherigen Genehmigungsverfahrens nach $\S \S 23,24$ Abs. 1 StrlSchV. Der Anwendungsbereich bleibt grundsätzlich gleich. Alle Forschungsvorhaben, bei denen ionisierende Strahlung zu therapeutischen Zwecken angewendet werden soll, bedürfen auch zukünftig der Genehmigung durch das BfS, § 185 Abs. 1 Nr. 1 und 2 StrlSchG. Klarstellend wurde in $\S 31$ Abs. 1 Satz 2 StrlSchG nunmehr geregelt, dass eine Genehmigung auch dann erforderlich ist, wenn eine wesentliche Abweichung von der genehmigten Exposition erfolgen soll. Wesentlich ist die Änderung dann, wenn die Änderung eine Genehmigungsvoraussetzung oder den Strahlenschutz der Teilnehmer betrifft.

Die Voraussetzungen, um die Genehmigung zu erhalten, werden ebenfalls gleich bleiben. Insbesondere wird sich der Umfang der vorzulegenden Unterlagen nicht ändern. ${ }^{12}$

\section{Prüfungsfristen}

Eine wirkliche Neuerung findet sich in $\S 31$ Abs. 3 StrlSchG. Im Laufe des Gesetzgebungsverfahrens konnte der Bundesrat durchsetzen, dass sowohl bei der Genehmigung als auch bei der Anzeige fixe und verbindliche Prüfungsfristen für die Bearbeitung durch das BfS eingefügt wurden. ${ }^{13}$

Formale Prüfung Die Prüfung durch das Bundesamt wird zukünftig zweistufig erfolgen. In einem ersten Prüfungsschritt, der 21 Kalendertage ab Eingang des Genehmigungsantrags nicht überschreiten soll, prüft das BfS die Vollstän-

\footnotetext{
11 BT-Drs. 18/11241, S. 219.

12 BT-Drs. 18/11241, S. 188.

13 BT-Drs. 18/12151 vom 26.04.2017 in Reaktion auf Stellungnahme des Bundesrates vom 10.03.2017, BR-Drs. 86/17, S. 13; Empfehlungen der Ausschüsse zu Punkt 15 der 954. Sitzung des Bundesrates am 10. 03.2017, BR-Drs. 86/1/7, S. 15.
}

digkeit der Unterlagen (formale Prüfung). Bei Unvollständigkeit soll das BfS den Antragsteller zur Vervollständigung innerhalb von weiteren 21 Kalendertagen auffordern. Diese erste Prüfungsphase kommt damit ohne fixe Fristen aus. Auch ist es möglich, dass es mehrere Runden dieser ersten Prüfungsphase geben wird. Dies beispielsweise, wenn sich bei der an die Nachlieferung von Unterlagen anschlieBenden Prüfung auf Vollständigkeit erneut Mängel zeigen sollten.

Inhaltliche Prüfung Wenn die Unterlagen vollständig sind, schließt sich unmittelbar die zweite Prüfungsphase an (inhaltliche Prüfung). Hierbei handelt es sich um eine fixe Frist, die zunächst 90 Kalendertage beträgt. Innerhalb dieser Frist ist das BfS verpflichtet, über die Genehmigung zu entscheiden. Das BfS kann diese Frist einmalig um weitere 90 Kalendertage verlängern, wenn dies wegen der Schwierigkeit der Prüfung im Einzelfall erforderlich ist. Die Verlängerung ist rechtzeitig mitzuteilen und zu begründen. Nach Ablauf der verlängerten Frist gilt die Genehmigung als erteilt (Genehmigungsfiktion), wenn das BfS nicht innerhalb dieser Frist über den Antrag entschieden hat. Bei der Verlängerung handelt es sich ausweislich der Gesetzessystematik um eine Ausnahmeregelung. Keinesfalls kann das BfS mit der Begründung, radioonkologische Forschungsvorhaben seien per se komplex, die Verlängerung zum Standard erheben. Vielmehr muss es sich um eine relative Schwierigkeit im Vergleich zu sonstigen radioonkologischen Studien handeln, nicht um eine absolute. Schließlich dürfen auch personelle Engpässe beim BfS nicht herangezogen werden, da diese nicht eine ,Schwierigkeit der Prüfung" zu begründen vermögen.

Diese starre Fristenregelung in Kombination mit der Genehmigungsfiktion bei Nichtbearbeitung ist eine echte Neuerung im Strahlenschutzrecht. Bisher ungeklärt ist, wie diese Regelungen in der Praxis umzusetzen sind. So stellt sich die Frage, wie umfangreich die Begründung des BfS sein muss, um die verlängerte Prüfungsfrist rechtmäßig bestimmen zu können. Die Gesetzesbegründung verweist hier auf eine vergleichbare Norm aus dem allgemeinen Verwaltungsrecht. ${ }^{14}$ Auch ist zu klären, wie diese inhaltliche Prüfung innerhalb von 90 (+90) Kalendertagen von der formalen Prüfung innerhalb von $21(+21)$ Kalendertagen $\mathrm{zu}$ trennen ist. Wie ist etwa vorzugehen, wenn nach Beginn der inhaltlichen Prüfung erkannt wird, dass nunmehr dennoch Unterlagen fehlen?

\footnotetext{
14 BT-Drs. 18/12151 vom 26.04.2017, S. 20 mit Verweis auf das „Vorbild des $\S 42 \mathrm{a}$ VwVfG“.
} 


\section{Rechtliche Folge der Genehmigungsfiktion}

Im Falle der Nichtbearbeitung innerhalb der (verlängerten inhaltlichen Prüfungsfrist) gilt die beantragte Genehmigung als durch einen fingierten Verwaltungsakt erteilt. Fraglich ist, ob dem BfS der gesamte 90- bis 180-Tage-Zeitraum zur Prüfung zur Verfügung steht oder ob innerhalb dieser Frist auch die Bekanntgabe der Entscheidung erfolgen muss. Während die Gesetzesbegründung zum als „Vorbild“ bezeichneten $\S 42 \mathrm{a}$ VwVfG ausführt, dass die Entscheidung innerhalb der Frist bekannt gegeben werden muss, führt das Bayerische Landessozialgericht (LSG) zum weitgehend vergleichbaren $\S 13$ Abs. 3a SGB V aus, dass die Behörde die gesamte dort genannte Frist zur Prüfung zur Verfügung hat, ${ }^{15}$ mithin die Fiktion erst nach Ablauf einer ebenfalls fiktiven Zustellfrist von 3 Tagen nach $\S 41$ Abs. 2 $\mathrm{VwVfG}^{16}$ beginnt.

Die Verlängerung der Prüfungsfrist ist nur zulässig, wenn dies rechtzeitig bekanntgegeben und begründet wurde. Die Begründung ist gerichtlich auf ihre Tragfähigkeit überprüfbar. Bei einer „unbegründeten“, d. h. nicht hinreichend begründeten Verlängerung, greift nach hiesiger Auffassung die Genehmigungsfiktion, da andernfalls das Erfordernis einer Begründung leerliefe. ${ }^{17}$

Rechtlich ist bei Eintritt der Genehmigungsfiktion die Situation so zu beurteilen, als hätte das BfS das Forschungsvorhaben im beantragen Umfang genehmigt. Mit der Studie kann daher - sofern die etwaig erforderlichen AMGoder MPG-rechtlichen Voraussetzungen erfüllt sind, unmittelbar begonnen werden. Im Sinne des sichersten Wegs sollte der Antragsteller jedoch nach Ablauf der (verlängerten) inhaltlichen Prüfungsfrist noch die 3-Tages-Fiktion des $\S 41$ Abs. 2 VwVfG abwarten, bevor er mit der Studie beginnt. Voraussetzung für die Genehmigungsfiktion ist, dass der Antrag „fiktionsfähig“ ist. Das bedeutet, dass sich der Inhalt der fingierten Genehmigung aus dem Antrag in Verbindung mit den einschlägigen Genehmigungsvorschriften hinreichend bestimmen lassen muss. ${ }^{18}$ In Anwendung des $\S 42 \mathrm{a}$ Abs. 3 VwVfG kann sich der Antragsteller den Eintritt der Genehmigungsfiktion von der Behörde schriftlich bestätigen lassen, um auf diesem Weg ein behördliches Schriftstück in den Händen halten zu können.

\footnotetext{
15 Siehe die Entscheidung des Bayerischen LSG, Urt. v. 31.01.2017 L 5 KR 471/15 - RN 38, zum insoweit vergleichbaren $\S 13$ Abs. 3a SGB 5.

16 Die 3-Tage-Fiktion gilt im Verwaltungsrecht nach $\S 41$ Abs. 2 S. 1 VwVfG für einen einfachen Brief.

17 So auch BSG zum insoweit vergleichbaren $\S 13$ Abs. 3a SGB V, der allerdings diese Rechtsfolge auch explizit in seinem Satz 6 aufführt, Urt. v. 08.03.2016 - B 1 KR 25/15 R - RN 20.

18 Dies ergibt sich aus der Gesetzesbegründung das für die hier relevante Fiktion als Vorbild genannte Regelung des § 42a VwVfG, BTDrs. 16/10493, S. 16.
}

$\mathrm{Da}$ es sich bei dieser fiktiven Genehmigung um eine „echte" Genehmigung handelt, finden auf sie die gleichen Rechtsfolgen Anwendung, wie auf einen tatsächlich ergangenen zustimmenden Verwaltungsakt. ${ }^{19}$ Insbesondere wirkt die Fiktion nicht auf die Rechtsmäßigkeit. Es gelten für den fiktiven Genehmigungsbescheid somit die gleichen Regeln zu Nichtigkeit, Rücknahme, Widerruf oder Erledigung wie für einen normalen Verwaltungsakt; jedoch kann das Fehlen der Entscheidung innerhalb der Prüfungsfrist regelmäßig nicht die Rücknahme oder den Widerruf der fingierten Genehmigung rechtfertigen, da andernfalls die Fiktion leerliefe. ${ }^{20}$ Bei der im Rahmen dieses möglichen Behördenhandelns erforderlichen Ermessensausübung ist das schutzwürdige Vertrauen des Antragstellers besonders zu berücksichtigen; so kann der Verhältnismäßigkeitsgrundsatz es gebieten, dass die fingierte Genehmigung nicht aufgehoben wird, sondern allein mit einschränkenden Nebenbestimmungen versehen wird. ${ }^{21}$ Dies dürfte insbesondere in den Fällen von Bedeutung sein, in denen mit der Durchführung der Studie auf Basis der Genehmigungsfiktion bereits begonnen wurde. In diesen Fällen haben auch die Teilnehmer an der Studie (Person im Sinne des $\S 2$ Abs. 8 Nr. 2 StrlSchG) ein schutzwürdiges Interesse an deren Fortführung.

\section{Ermessensentscheidung vs. gebundene Entscheidung}

Bei der Entscheidung des BfS über die Genehmigung des Forschungsvorhabens handelt es sich ausweislich der Gesetzesbegründung um eine Ermessensentscheidung, d.h., selbst wenn alle in $\S 31$ Abs. 4 aufgeführten Unterlagen und Voraussetzungen vorliegen, hat das BfS nach pflichtgemäßem Ermessen zu entscheiden. Dies begründet der Gesetzgeber mit einem Vergleich zum Wortlaut des § 25 Abs. 3 StrlSchG. Während es dort heißt, „die Behörde hat die Genehmigung zu erteilen, wenn“", heißt es bei $\S 31$ Abs. 4, die „Behörde darf die Genehmigung nur erteilen, wenn“. Aufgrund des abschließenden Charakters des Voraussetzungskatalogs des $\S 31$ Abs. 4 bleibt jedoch faktisch kein oder nur ein sehr geringer Raum für ein Ermessen, wenn die dort genannten Voraussetzungen erfüllt sind. Dass der Gesetzgeber aber einen geringen Ermessenspielraum vorsieht, ergibt sich auch durch einen Vergleich mit der Regelung des $\S 31$ Abs. 6 StrlSchG, der die Genehmigung von Multicenterstudien beinhaltet. Dort heißt es,

\footnotetext{
19 S. oben.

${ }^{20}$ So die Gesetzesbegründung für die hier relevante Fiktion als Vorbild genannte Regelung des $\S 42 \mathrm{a}$ VwVfG, BT-Drs. 16/10493, S. 16.

${ }^{21}$ S. oben.
} 
so erteilt die zuständige Behörde eine umfassende Genehmigung für alle Einrichtungen, für die die Voraussetzungen nach Absatz 4 Nummer 6 und 8 erfüllt sind.

Daraus folgt, dass eine Studie, die als Einzelzentrumsstudie genehmigungsfähig wäre, ohne Ermessenspielraum der Behörde als Multicenterstudie genehmigt werden muss, wenn die einzelnen Einrichtungen die personellen und sachlichen Voraussetzungen erfüllen.

\section{Zeitaufwand beim Genehmigungsverfahren (nach § 31 StrlSchG)}

Bei einer Addition der beim BfS erforderlichen Schritte bis zur Erteilung einer (fingierten) Genehmigung durch das BfS vergehen maximal $222(+3)$ Tage. Die formale Prüfung benötigt 21-42 Tage und die inhaltliche Prüfung 90-180 Tage. Hinzu kommt ggf. die 3-Tages-Fiktion des $\S 41$ Abs. 2 VwVfG bei einer fiktiven Genehmigung. Zu beachten ist jedoch, dass beim Genehmigungsverfahren nach $\S 31$ StrlSchG die Einholung der Genehmigung der Ethikkommission nicht parallel, sondern vorgeschaltet erfolgen muss. Hierfür sind zusätzlich 60 Tage anzusetzen ( $\$ 36$ Abs. 2 StrlSchG). Insgesamt beläuft sich damit die maximal benötigte Zeitspanne zwischen Antrag bei der Ethikkommission und finaler Genehmigung durch das BfS auf $282(+3)$ Tage oder auf ein gutes Dreivierteljahr.

\section{Anzeigeverfahren (nach § 32 ff., 37 StrISchG)}

Forschungsvorhaben, bei denen die Anwendung ionisierender Strahlen allein begleitdiagnostischen Zwecken bei volljährigen Patienten (,kranken Menschen“) vorbehalten ist, können im sog. Anzeigeverfahren nach $\S \S 32$ ff. StrlSchG dem BfS bekannt gegeben werden. Der Anzeige sind die in $\S 32$ Abs. 2 StrlSchG im Einzelnen aufgeführten Unterlagen beizufügen. Auch beim Anzeigeverfahren wird zwischen der formalen und der inhaltlichen Prüfung differenziert werden.

\section{Formale Prüfung (§ 33 Abs. 1 StrlSchG)}

Das BfS prüft nach $\S 33$ StrlSchG, ob die Anzeige vollständig ist. Ist dies der Fall, erhält der Anzeigende eine entsprechende Bestätigung innerhalb von 14 Kalendertagen. Andernfalls erfolgt in derselben 14-Tage-Frist die einmalige Aufforderung, die vom BfS aufzuführenden Mängel der Anzeige innerhalb einer Frist von weiteren 10 Tagen zu beheben. Nach Zugang der ergänzenden Unterlagen prüft das BfS die Vollständigkeit der Anzeige abschließend und informiert den Anzeigenden. Für den Fall, dass die Behörde eine negative Mitteilung macht, stehen dem Anzeigenden die üblichen verwaltungsrechtlichen Rechtsbehelfe zu. ${ }^{22}$

\section{Inhaltliche Prüfung ( $\$ 33$ Abs. 2 StrlSchG)}

Sofern die Anzeige vollständig ist, prüft das BfS nach $\S 33$ Abs. 2 StrlSchG in weiteren 28 Kalendertagen das Forschungsvorhaben inhaltlich. Bei Einwänden erfolgt auch in diesem zweiten Prüfungsschritt eine mit Gründen versehene einmalige Aufforderung zur Überarbeitung der Anzeige, für die das Gesetz dem Anzeigenden 21 Kalendertage zubilligt. ${ }^{23}$ Nach Eingang der Änderungen/Ergänzungen durch den Anzeigenden schließt das BfS die Prüfung innerhalb von weiteren 21 Kalendertagen ab.

Wenn das BfS innerhalb dieses Prüfungszeitraums zu dem Ergebnis kommt, dass die Anforderungen des $\S 32$ Abs. 2-4 StrlSchG (endgültig) nicht erfüllt sind, kann es die angezeigte Anwendung ionisierender Strahlen untersagen ( 34 Abs. 1 StrlSchG). Nach Ablauf dieser Prüfungsfrist kann eine Untersagung nur noch erfolgen, wenn die Anforderungen nach § 32 Abs. 2-4 StrlSchG nicht oder nicht mehr erfüllt sind und keine Abhilfe in angemessener Zeit geschaffen wird, dem BfS keine zustimmende Stellungnahme einer Ethikkommission in angemessener Zeit nach Fristsetzung vorgelegt wird oder sonstige erhebliche oder wiederholte Rechtsverstöße vorliegen und keine Abhilfe in angemessener Zeit erfolgt. Welche Zeitspanne (noch) angemessen ist, bestimmt das Gesetz nicht. Bei einem Vergleich mit den Zeitvorgaben des $\S 32$ StrlSchG dürfte es sich - je nach Schwere des Problems - aber um einen Zeitraum von wenigen Tagen bis maximal 3 Wochen handeln. Bei allen Entscheidungen des BfS im Rahmen des Anzeigeverfahrens handelt es um Ermessensentscheidungen. Die Ausübung dieses Ermessens und insbesondere die behaupteten Grundlagen der Ermessensentscheidung sind jedoch gerichtlich überprüfbar.

\section{Zeitaufwand beim Anzeigeverfahren nach § 32 StrlSchG}

Wenn das BfS nicht innerhalb der Frist nach $\S 32$ Abs. 2 die Anwendung nach $\S 34$ Abs. 1 StrlSchG untersagt hat, ein zustimmendes Votum der Ethikkommission vorliegt und entweder die Frist nach $\S 32$ Abs. 2 StrlSchG (inhaltliche Prüfung der Anzeige) verstrichen oder das BfS explizit auf das Ausschöpfen der Frist verzichtet hat, darf mit dem For-

\footnotetext{
22 BT-Drs. 18/11241, S. 204 - Der Gesetzgeber geht von einer Zunahme der Anzahl der Untersagungen aus, da nunmehr allein eine alleinige Nachbesserung der Antragsunterlagen zulässig sein wird. Entsprechend wird auch mit einer Zunahme der Widerspruchsverfahren gerechnet, zu deren Bearbeitung der Gesetzgeber eine halbe juristische Fachkraft beim BfS einkalkuliert.

${ }^{23}$ Auch hier bestehen die allgemeinen verwaltungsrechtlichen Rechtsbehelfe.
} 
schungsvorhaben begonnen werden. Da beim Anzeigeverfahren das positive Votum der Ethikkommission nicht Voraussetzung für die inhaltliche Prüfung des BfS sein wird, können diese Verfahren parallel laufen, was zu einer erheblichen Zeitersparnis führen kann. Die formale Prüfung nach $\S 32$ Abs. 1 StrlSchG beträgt zwischen 14 und 36 Kalendertagen. Für die sich anschließende inhaltliche Prüfung bewilligt das Gesetz dem BfS zwischen 28 und 70 Kalendertagen. Im schnellstmöglichen Falle einer unproblematischen und von Beginn an vollständigen Anzeige kann das Verfahren in maximal 42 Tagen (14 Tage formale und 28 Tage inhaltliche Prüfung) abgeschlossen werden, bei explizitem Verzicht des BfS auf Ausschöpfung der Prüfungsfrist auch schneller. In diesem Fall würde sich der 60-Tage-Zeitraum für das Votum der Ethikkommission nach $\S 36$ Abs. 2 StrlSchG als längster Zeitraum darstellen, der aber aufgrund der Möglichkeit einer parallelen Bearbeitung damit auch insgesamt den möglichen zeitlichen Maximalaufwand bei einem unproblematischen Vorhaben darstellt. ${ }^{24}$

\section{Verordnungsermächtigung für Detailfragen (nach § 37 StrISchG)}

Das Strahlenschutzgesetz verhält sich nicht zu den Detailfragen der Exposition im Rahmen und in der Durchführung von Forschungsvorhaben. Diese sollen im Rahmen einer noch zu schaffenden Rechtsverordnung der Bundesregierung mit Zustimmung des Bundesrates erfolgen. Dass der Bundesrat hier ein wichtiges Korrektiv sein kann, zeigt die Aufnahme der Genehmigungsfiktion in $\S 31$ StrlSchG (Genehmigungsverfahren), die von der Bundesregierung und dem Bundestag ursprünglich nicht vorgesehen war. Eine nichtabschließende Aufzählung der Aspekte, die Gegenstand der Rechtsverordnung sein können, findet sich in $§ 37$ Abs. 1 Satz 2 Nr. 1 bis 8 StrlSchG. Von besonderer Bedeutung aus Sicht der Radioonkologie sind die in Nr. 5 genannten Grenzwerte und die Maßnahmen zu deren Einhaltung sowie die in Nr. 8 genannten Mitteilungs- und Berichtspflichten.

\section{Grenzwerte für Probanden (§ 37 Abs. 1 Satz 2 Nr. 5 StrlSchG)}

Ausweislich der Gesetzesbegründung geht es hier insbesondere um Grenzwerte für gesunde Probanden und damit um ein Kollektiv, dass im Rahmen radioonkologischer Studien nicht relevant ist. Grenzwerte bei erkrankten Probanden/ Patienten sind unzulässig.

\footnotetext{
${ }^{24}$ Selbstverständlich muss auch die Ethikkommission den 60-TageZeitraum nicht vollständig ausschöpfen, sodass eine noch weitergehende Beschleunigung denkbar ist.
}

Mitteilungs- und Berichtspflicht ( $\$ 37$ Abs. 1 Satz 2 Nr. 8 StrISchG)

Laut Gesetzesbegründung ist hier an Regelungen gedacht, wie sie bisher in $\S 89$ StrlSchV enthalten sind. Nicht verwechselt werden dürfen diese Mitteilungs- und Berichtspflichten mit den neu eingeführten Meldepflichten bei Vorkommnissen nach $\S 90$ StrlSchG, die unabhängig von Forschungsvorhaben bestehen werden und ebenfalls noch durch eine Rechtsverordnung zu konkretisieren sind.

\section{Inkrafttreten der neuen Vorschriften und Übergangsvorschriften}

Die für die Forschung und Heilbehandlung relevanten Vorgaben des neuen StrlSchG treten gemäß Art. 32 des Gesetzes zur Neuordnung des Rechts zum Schutz vor der schädlichen Wirkung ionisierender Strahlung zum 01.01.2019 in Kraft. ${ }^{25}$ Gemäß $§ 205$ Abs. 1 bis 4 StrlSchG gelten für die medizinische Forschung nach den $\S \S 31,32$ StrlSchG folgende Übergangsvorschriften:

- $\S 205$ Abs. 1 StrlSchG: Forschungsvorhaben, die bis zum 31.12.2018 nach §§ 23, 24 Abs. 1 StrlSchV (,normale“ Genehmigung nach altem Recht) genehmigt worden sein werden, gelten als Genehmigung nach $\S 31$ mit allen $\mathrm{Ne}$ benbestimmungen fort.

- $\S 205$ Abs. 2 StrlSchG: Forschungsvorhaben, die bis zum 31.12.2018 nach $\S \S 23,24$ Abs. 2 StrlSchV (,vereinfachte" Genehmigung nach altem Recht) genehmigt worden sein werden, gelten als Anzeige nach $\S 32$ StrlSchG fort.

- $\S 205$ Abs. 3 StrlSchG: Vor dem 31.12.2018 begonnene Genehmigungsverfahren nach $\S \S 23,24$ Abs. 2 StrlSchV (,vereinfachte“"Genehmigung nach altem Recht) werden nach altem Recht abgeschlossen. Nach erteilter Genehmigung gilt diese als Anzeige nach $\S 32$ StrlSchG.

- § 205 Abs. 4 StrlSchG: Registrierungen von Ethikkommissionen nach $\S 92 \mathrm{StrlSchV}$ gelten als Registrierung nach $\S 36$ Abs. 1 StrlSchG.

Es fällt auf, dass keine Übergangsvorschrift für nichtabgeschlossene Genehmigungsverfahren nach $\S \S 23$, 24 Abs. 1 StrlSchV (,normale“ Genehmigungen nach altem Recht) existiert. Das bedeutet, dass vor dem 31.12.2018 begonnene, aber noch nicht abgeschlossene Genehmigungsverfahren für entsprechende Forschungsvorhaben (therapeutischer Einsatz ionisierender Strahlen) mit dem Jahreswechsel 2018/2019 obsolet werden und ab dem 01.01.2019 als Genehmigungsverfahren nach $\S 31$ StrlSchG neu beantragt werden müssen. Aufgrund der bekannten Dauer für solche Genehmigungen nach $\S \S 23,24$ Abs. 1 StrlSchV

25 BGBl. vom 03.07.2017 S. 1966-2067, 2067. 
ist daher den interessierten Kreisen von der Stellung eines Antrags auf Genehmigung im Vorfeld des Jahreswechsels abzuraten, wenn das Verfahren absehbar nicht vor dem Jahreswechsel abgeschlossen sein werden wird. Mangels expliziter Regelung zur Fortgeltung erteilter zustimmender Voten der Ethikkommissionen ist in entsprechender Anwendung des $\S 205$ Abs. 4 StrlSchG (Fortgeltung der Registrierung von Ethikkommissionen) davon auszugehen, dass jedenfalls dieses Votum auch nach neuem Recht Bestand haben wird. Dafür spricht auch, dass sich der Prüfungsumfang der Ethikkommissionen in Bezug auf die strahlenschutzrechtlichen Aspekte nicht verändern soll. ${ }^{26}$

\section{Einhaltung ethischer Richtlinien}

Interessenkonflikt T. Nölling war verschiedentlich im Auftrag der DEGRO zum Thema Forschung in der Radioonkologie tätig.

Ethische Standards Dieser Beitrag beinhaltet keine von den Autoren durchgeführten Studien an Menschen oder Tieren.

${ }^{26}$ BT-Drs. 18/11241, S. 276. 